\title{
Ectodermal dysplasia syndrome
}

INSERM

\section{Source}

INSERM. (1999). Orphanet: an online rare disease and orphan drug data base.

Ectodermal dysplasia syndrome. ORPHA:79373

The term "ectodermal dysplasia" defines a heterogeneous group of heritable disorders of the skin and its appendages characterized by the defective development of two or more ectodermal derivatives, including hair, teeth, nails, sweat glands and their modified structures (i.e. ceruminous, mammary and ciliary glands). The spectrum of clinical manifestations is wide and may include additional manifestations from other ectodermal, mesodermal and endodermal structures. 